\title{
Minimum cost design for fire resistance of welded steel structures
}

\author{
József Farkas ${ }^{1}$ and Károly Jármai ${ }^{2}$ \\ University of Miskolc, H-3515 Miskolc, Hungary \\ ${ }^{1}$ Professor emeritus, Dr.sci.techn.Dr.h.c. altfar@uni-miskolc.hu \\ ${ }^{2}$ Professor, Dr.sci.techn. altjar@uni-miskolc.hu
}

\begin{abstract}
The optimum design is applied to cost minimization of two types of welded steel structures in fire. Both unprotected and protected structures are investigated. The compressed rod of welded square box crosssection is designed to overall and local buckling. The bent beam of welded box section should fulfil the stress, deflection and local buckling constraints. The cost function consists of cost of material, assembly, welding, painting and fire protection. In the unprotected case the critical temperature method is used with formulae given in Eurocode 3. At both structures the protected one is cheaper than the unprotected. This difference is caused by the significant difference in thicknesses.
\end{abstract}

\section{Keywords}

Structural optimization, welded structure, fire resistant design, stability, cost calculation

\section{INTRODUCTION}

Requirements for modern load-carrying structures are the safety, fitness for production and economy. In the optimum design procedure the safety and fitness for production are guaranteed by fulfilling of design and fabrication constraints, and the economy is achieved by minimization of a cost function.

It is possible to design a lot of structural versions. The most suitable version can be selected by cost comparison. For the purpose of economic design of welded steel structures a relatively simple cost calculation method is developed $[1,2,3]$. The cost function consists of cost of material, assembly, welding and painting. 
Since the fire resistance of steel structures needs protection, the cost of various protection methods is also calculated using numerical data from industry.

The search for better solutions is performed by change of structural characteristics such as material, type of structure, profiles, main dimensions, fabrication technology and connections.

In general, the optimum design needs the solution of a constrained minimization of one or more objective nonlinear multivariable functions. Therefore the problems can only be treated numerically and the results are valid in general. In spite of this, when the numerical data of problems are selected as near as possible to industrial application, the results are very useful for designers to find the most economic and competitive structural versions.

In our research work we have worked out a lot of numerical problems of various metal structures. Our aim is to show how to apply the economic design for fire-resistant welded steel structures. The catastrophic damages and failures show that steel structures are very sensitive to high temperatures. Therefore special design rules have been elaborated in relevant Eurocodes $[4,5,6]$, which are applied in the present paper.

Two numerical problems are solved as follows: (1) a centrally compressed rod of welded square box cross-section, (2) a welded box beam loaded in bending and shear.

Costs of unprotected and protected versions are compared to each other. Since only optimized versions can be compared, the both versions are optimized for minimum cost.

\section{THE CRITICAL TEMPERATURE METHOD}

Figure 1 shows the temperature versus time for fire gas and for a steel structure. The gas temperature can be calculated as

$$
\Theta_{g}=20+345 \log \left(\frac{8 T}{60}+1\right)
$$

where $T$ is the time in $s$.

The temperature of steel structure in a time interval is given by

$$
\Delta \Theta_{a}=\frac{A_{m}}{V} \frac{h_{\text {netd }}}{c_{a} \rho_{m}} \Delta T
$$

where $c_{a}$ is the specific heat of steel,

$$
c_{a}=425+7.73 \times 10^{-1} \Theta_{a}-1.69 \times 10^{-3} \Theta_{a}^{2}+2.22 \times 10^{-6} \Theta_{a}^{3}
$$

$\rho_{m}$ is the unit mass of steel, $A_{m} / V$ is for rods of constant cross-section the ratio of perimeter/cross-section area, for a square box section

$$
A_{m} / V=1 / t
$$

The design value of the net heat flux per unit area is 


$$
h_{\text {netd }}=h_{\text {netc }}+h_{\text {netr }}
$$

where the net convection heat flux is

$$
h_{\text {netc }}=25\left(\Theta_{g}-\Theta_{a}\right)
$$

and the net radiative heat flux is

$$
h_{\text {netr }}=0.8 \times 5.67 \times 10^{-8}\left[\left(\Theta_{g}+273\right)^{4}-\left(\Theta_{a}+273\right)^{4}\right]
$$

$5.67 \times 10^{-8}$ is the Boltzmann constant.

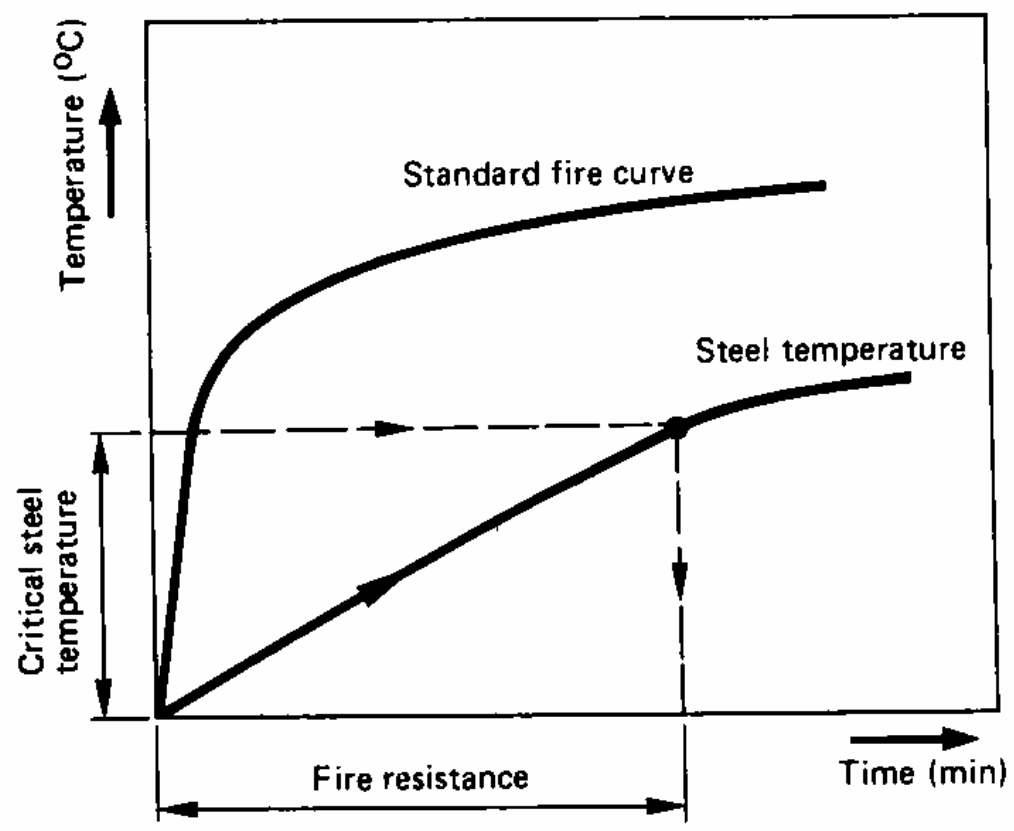

Figure 1. The critical temperature method

The critical temperature is given by

$$
\Theta_{c r}=39.19 \ln \left(\frac{1}{0.9674 \mu_{0}^{3.833}}-1\right)+482
$$

where

$$
\mu_{0}=N_{f i} / N_{0}
$$

is the utilization factor, $N_{f i}$ and $N_{o}$ are the limiting compression forces in the case of fire and for ambient temperature, respectively.

The fire resistance time $R$ corresponding to the critical temperature can be obtained by step-by-step using eqns. (1)-(9). Since until $600^{\circ} \mathrm{C}$ the parameters in eqn. (2). can be approximated by three linear intervals, we use intervals of

$$
\Theta_{a 1}=\Theta_{c r} / 3, \Theta_{a 2}=2 \Theta_{c r} / 3, \Theta_{a 3}=\Theta_{c r}
$$

In this case the final $R=\sum R_{i}$ is calculated by three iterations using a MathCad program. 


$$
\Delta R_{i}=\frac{\Theta_{a i} c_{a i} \rho_{m}}{6 \times 10^{4} h_{\text {netdi }}}, i=1,2,3
$$

\section{A CENTRALLY COMPRESSED ROD WITH PINNED ENDS OF WELDED SQUARE BOX} CROSS-SECTION
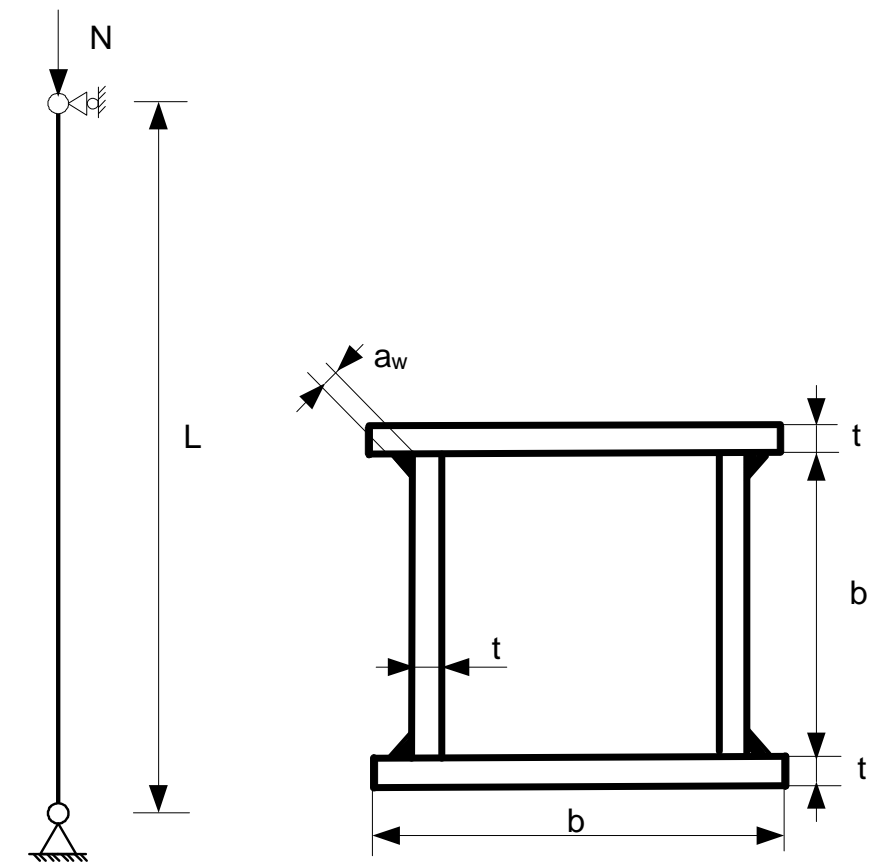

Figure 2. Compressed rod of welded square box section

\subsection{Overall buckling constraint for ambient temperature}

$$
\begin{aligned}
& N \leq N_{0} \\
& N_{0}=\chi f_{y} A
\end{aligned}
$$

the buckling factor

$$
\chi=\frac{1}{\phi+\sqrt{\phi^{2}-\bar{\lambda}^{2}}}, \phi=\frac{1}{2}\left[1+\alpha(\bar{\lambda}-0.2)+\bar{\lambda}^{2}\right]
$$

where

$$
\bar{\lambda}=\frac{\lambda}{\lambda_{E}}, \lambda=\frac{L}{r}, r=\sqrt{\frac{I}{A}}, \lambda_{E}=\pi \sqrt{\frac{E}{f_{y}}}
$$

In the case of a square box section

$$
\begin{aligned}
& A=4 b t \\
& I=\frac{2 b^{3} t}{3}+\frac{b t^{3}}{6}
\end{aligned}
$$


For fire design $\alpha=0.49$.

\subsection{Overall buckling constraint in fire}

$$
\begin{aligned}
& N \leq N_{f i . t} \\
& N_{f i . t}=\chi_{f i} A k_{y \Theta i} f_{y} / \gamma_{M f i}
\end{aligned}
$$

$\gamma_{M f i=1}$

$$
\begin{aligned}
& \chi_{f i}=\frac{1}{\varphi_{\Theta}+\sqrt{\varphi_{\Theta}^{2}-\bar{\lambda}_{\Theta}^{2}}}, \varphi_{\Theta}=\frac{1}{2}\left(1+\alpha \bar{\lambda}_{\Theta}+\bar{\lambda}_{\Theta}^{2}\right) \\
& \alpha=0.65 \sqrt{\frac{235}{f_{y}}, \bar{\lambda}_{\Theta}}=\bar{\lambda} \sqrt{\frac{k_{y \Theta i}}{k_{E \Theta i}}}
\end{aligned}
$$

Factors of $k_{y \Theta i}$ and $k_{E \Theta i}$ can be approximated by linear intervals of

$$
\begin{aligned}
& k_{y \Theta 0}=1 \quad \text { if } 20^{\circ} \mathrm{C}<\Theta_{a}<400^{\circ} \mathrm{C} \\
& k_{y \Theta 1}=\frac{500-\Theta_{a}}{100} 0.22+0.78 \text { if } 400^{\circ} \mathrm{C}<\Theta_{a}<500^{\circ} \mathrm{C} \\
& k_{y \Theta 2}=\frac{600-\Theta_{a}}{100} 0.31+0.47 \text { if } 500^{\circ} \mathrm{C}<\Theta_{a}<600^{\circ} \mathrm{C}
\end{aligned}
$$

and

$$
\begin{array}{ll}
k_{E \Theta 0}=1 & \text { if } 20^{\circ} \mathrm{C}<\Theta_{a}<100^{\circ} \mathrm{C} \\
k_{E \Theta 1}=\frac{500-\Theta_{a}}{400} 0.4+0.6 & \text { if } 100^{\circ} \mathrm{C}<\Theta_{a}<500^{\circ} \mathrm{C} \\
k_{E \Theta 2}=\frac{600-\Theta_{a}}{100} 0.29+0.31 & \text { if } 500^{\circ} \mathrm{C}<\Theta_{a}<600^{\circ} \mathrm{C}
\end{array}
$$

\subsection{Local buckling constraint}

For ambient temperature

$$
b / t \leq 42 \varepsilon, \varepsilon=\sqrt{235 / f_{y}}
$$

For fire Eurocode 3 proposed a decreased value of

$$
b / t \leq 0.8 x 42 \varepsilon=33.6 \varepsilon
$$

According to the experiments of Knobloch [7] and calculations of Heidarpour \& Bradford [8]

$$
b / t \leq 0.6 \times 42 \varepsilon=25.2 \varepsilon
$$




\subsection{Cost function}

The general formula for the welding cost is as follows $[2,3,4]$ :

$$
K_{w}=k_{w}\left(C_{1} \Theta \sqrt{\kappa \rho V}+1.3 \sum_{i} C_{w i} a_{w i}^{n} C_{p i} L_{w i}\right)
$$

where $k_{w}[\$ / \mathrm{min}]$ is the welding cost factor, $C_{1}$ is the factor for the assembly usually taken as $C_{1}=1$ $\min / \mathrm{kg}^{0.5}, \Theta$ is the factor expressing the complexity of assembly, the first member calculates the time of the assembly, $\kappa$ is the number of structural parts to be assembled, $\rho V$ is the mass of the assembled structure, the second member estimates the time of welding, $C_{w}$ and $n$ are the constants given for the specified welding technology and weld type, $\mathrm{Cp}$ is the factor of welding position (for downhand 1, for vertical 2, for overhead 3).

Furthermore $C_{p i}$ is the factor for the welding position (download 1, vertical 2, overhead 3), $L_{w}$ is the weld length, the multiplier 1.3 takes into account the additional welding times (deslagging, chipping, changing the electrode).

Material cost

$$
K_{m}=k_{m} \rho V, V=A L, k_{m}=1.0 \$ / \mathrm{kg}
$$

Welding cost for 4 fillet welds of GMAW-C (Gas metal arc welding with $\mathrm{CO}_{2}$ ) [4]

$$
K_{w}=k_{w}\left(C_{1} \Theta_{c} \sqrt{\kappa \rho V}+1.3 C_{w} a_{w}^{2} L_{w}\right), k_{w}=1.0 \$ / \min : C_{1}=1.0 \mathrm{~min} / \mathrm{kg}^{0.5}
$$

The factor of complexity of assembly is $\Theta_{c}=2$, number of assembled parts is $\kappa=4$, fillet weld size $a_{w}=$ $0.3 t$, welding coefficient $C_{w}=0.3394 \times 10^{-3}$, length of welds $L_{w}=4 L$.

Painting cost

$$
K_{p}=k_{p} S, k_{p}=28.8 \times 10^{-6} \$ / \mathrm{mm}^{2}, S=4 b L
$$

Total cost

$$
K=K_{m}+K_{w}+K_{p}
$$

\subsection{Numerical data and results}

Centric compression force for fire $N=10^{7}[\mathrm{~N}]$. This load is calculated from the actual ones using a reduction factor $\eta_{f i}$. Rod length $L=6 \mathrm{~m}$. Yield stress of steel $f_{y}=235 \mathrm{MPa}$.

The optimization is performed by a systematic search using a MathCad program. Results are given in Tables 1 . and 2 . 
Table 1. Results for the unprotected structure for a fire resistance time $R=30 \mathrm{~min}$. Optimum is marked by bold letters

\begin{tabular}{ccccccc}
\hline$b \mathrm{~mm}$ & $t \mathrm{~mm}$ & $10^{-3} A$ & $K \$$ & $\Theta_{c r}{ }^{0} \mathrm{C}$ & $R \min$ & $10^{-7} N_{f i . T}[\mathrm{~N}]$ \\
\hline 500 & 38 & 76.00 & 5541 & 556 & 31.2 & 1.013 \\
500 & 37 & 74.00 & 5372 & 551 & 30.2 & 0.977 \\
$\mathbf{5 1 0}$ & $\mathbf{3 7}$ & $\mathbf{7 5 . 4 8}$ & $\mathbf{5 4 5 1}$ & $\mathbf{5 5 5}$ & $\mathbf{3 0 . 5}$ & $\mathbf{1 . 0 0 3}$ \\
520 & 36 & 74.88 & 5359 & 554 & 29.9 & 0.856 \\
530 & 35 & 74.20 & 5265 & 553 & 29.4 & 0.857 \\
\hline
\end{tabular}

Table 2. .Results for protected structure for fire resistance time $R=60 \mathrm{~min}$. Optimum is marked by bold letters. $K$ is the cost according to eqn. (35) without the cost of protection. The result in the last row does not fulfil the local buckling constraint

\begin{tabular}{cccccc}
\hline$b \mathrm{~mm}$ & $t \mathrm{~mm}$ & $10^{-3} A$ & $K \$$ & $10^{-7} N_{f i t T}[\mathrm{~N}]$ & $b / t$ \\
\hline 630 & 20 & 50.40 & 3385 & 1.012 & 31.5 \\
$\mathbf{6 6 0}$ & $\mathbf{1 9}$ & $\mathbf{5 0 . 1 6}$ & $\mathbf{3 3 5 7}$ & $\mathbf{1 . 0 1 4}$ & $\mathbf{3 4 . 7}$ \\
700 & 18 & 50.40 & 3361 & 1.028 & 38.9 \\
720 & 17 & 48.96 & 3271 & 1.003 & 42.4 \\
\hline
\end{tabular}

\subsection{Cost including protection}

The following approximate cost data are from Hungarian industry.

\section{(a) Intumescent paint „Polylack” [9]}

Cost factor $k_{p l}=60 \$ / \mathrm{m}^{2}$, superficies $: S=4 \times 0.66 \times 6=15.84 \mathrm{~m}^{2}$

$K_{p 1}=k_{p 1} S=950 \$$

$K_{1}=K-K_{p}+K_{p l}=3357-456+950=3851 \$$

Cost without protection $K=5451$, thus, the cost savings is $29 \%$.

\section{(b) fire resistant plasterboard „Rigips” of thickness 12.5 mm [10]}

Cost factor $k_{p 2}=5.0 \$ / \mathrm{m}^{2}, K_{p 2}=k_{p 2} S=79.0 \$$, labour cost $K_{L}=70 \$$

$K_{2}=3357-456+79+70=3050 \$$ 
Cost without protection $K=5451 \$$, thus, the cost savings is $44 \%$.

\section{A SIMPLY SUPPORTED UNIFORMLY LOADED WELDED BOX BEAM}

Optimum design of this structure is treated for four cases as follows: unprotected and protected beam with stress or deflection constraint. In Equations the following subscripts are used: unprotected stress constraint $\sigma$, unprotected deflection constraint $w$, protected stress constraint $\sigma 1$, protected deflection constraint $w 1$.
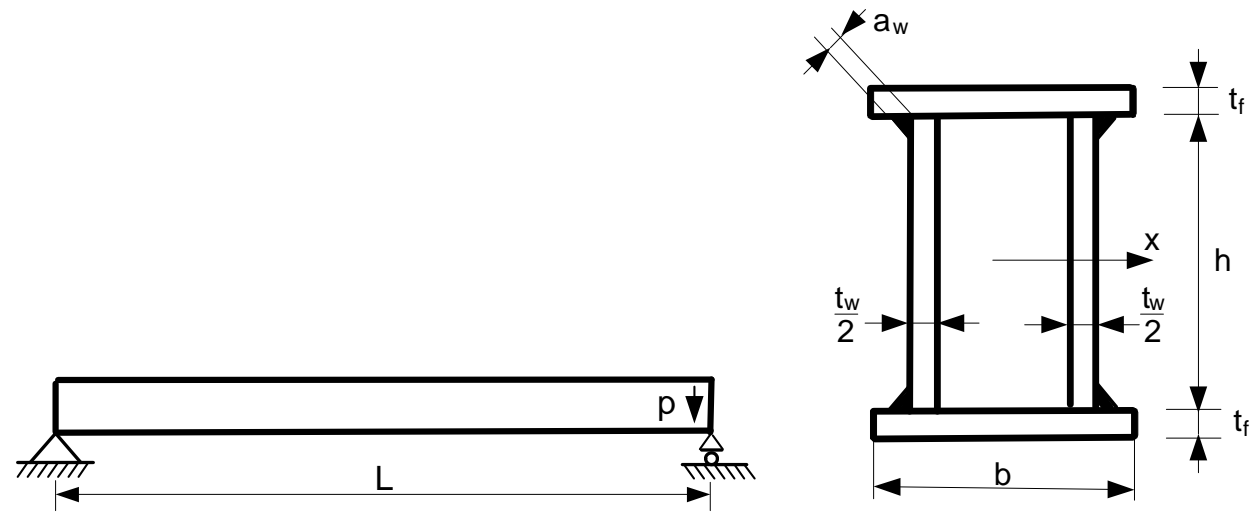

Figure 3. A simply supported welded box beam

\subsection{Optimum design}

It is sufficient to solve the optimization problem for minimum cross-section area instead of minimum cost, since the welding cost of four longitudinal fillet welds has no significant effect on the optimum beam dimensions.

The formulation of the optimum design of a box beam is as follows: find the optimum values of the dimensions $h, t_{w}, b, t_{f}$ to minimize the whole cross-section area

$$
A=h t_{w}+2 b t_{f}
$$

and fulfil the following constraints:

(a) stress constraint

$$
\begin{aligned}
& \sigma_{\max }=\frac{M}{W_{x}} \leq f_{y 1} \quad \text { or } \quad W_{x} \geq \frac{M}{f_{y 1}}=W_{0} \\
& I_{x}=\frac{h^{3} t_{w}}{6}+2 b t_{f}\left(\frac{h}{2}\right)^{2} ; W_{x}=\frac{I}{h / 2}=\frac{h^{2} t_{w}}{3}+b t_{f} h
\end{aligned}
$$

The bending moment is expressed as 


$$
M=p_{s} L^{2} / 8,
$$

the self mass of the sectorial plate is also taken into account, so

$$
p_{s}=1.5 p+1.1 \rho_{1} A, \rho_{1}=7.85 \times 10^{-5} \mathrm{~N} / \mathrm{mm}^{3} \text {. }
$$

(b) deflection constraint

$$
w_{\max }=\frac{C_{w}}{I} \leq w_{\text {adm }}=\frac{L}{\phi} ; C_{w}=\frac{5 p_{d} L^{4}}{384 E} ; \phi=300
$$

or

$$
I \geq I_{0}=\frac{5 p_{w} L^{4}}{384 E w_{a d m}}
$$

For the deflection constraint the load intensity is calculated without safety factors, thus

$$
p_{w}=p+\rho_{1} A
$$

(c) constraint on local buckling of webs

$$
\frac{h}{t_{w} / 2} \leq \frac{1}{\beta} ; \text { or } t_{w} \geq 2 \beta h
$$

where $1 / \beta=69 \varepsilon ; 1 / \beta_{f i}=69 \varepsilon \alpha_{f i}, \varepsilon=\sqrt{\frac{235}{f_{y}}}$

For unprotected beam $\alpha_{f i}=0.6$, for protected one $\alpha_{f i}=1$

(d) constraint for local buckling of compressed upper flange

$$
\frac{b}{t_{f}} \leq \frac{1}{\delta}=42 \varepsilon, \frac{1}{\delta_{f i}}=42 \varepsilon \alpha_{f i}, \quad \alpha_{f i}=0.6 \text { or } t_{f} \geq \delta b
$$

Considering the local buckling constraint as active the stress constraint can be written as

$$
W=\frac{\beta h^{3}}{3}+b t_{f} h \geq W_{0}
$$

substituting $b t_{f}$ from eqn. (36) into one obtains

$$
A=\frac{2 W_{0}}{h}+\frac{4 \beta h^{2}}{3}
$$

From the condition

$$
\frac{d A}{d h}=0
$$

one obtains the optimum value of $h$ from the stress constraint

$$
h_{\sigma}=\sqrt[3]{\frac{3 W_{0}}{4 \beta}}
$$

Similarly from deflection constraint 


$$
h_{w}=\sqrt[4]{\frac{3 I_{0}}{\beta}} ; I_{0}=\frac{\phi C_{w}}{L}
$$

The advantage of this optimization method is that the other characteristics of the optimum cross-section can be expressed by $h_{\sigma}$ or $h_{w}$. These characteristics are summarized in Table 3.

Table 3. Characteristics of optimum box sections

$$
\begin{array}{cc}
\text { Stress constraint } & \text { Deflection constraint } \\
h_{\sigma}=\sqrt[3]{0.75 W_{0} / \beta} & h_{w}=\sqrt[4]{3 I_{0} / \beta} \\
t_{w \sigma} / 2=\beta h_{\sigma} & t_{w w} / 2=\beta h_{w} \\
A_{\sigma}=4 \beta h_{\sigma}^{2}=\sqrt[3]{36 \beta W_{0}^{2}} & A_{w}=8 \beta h_{w}^{2} / 3=\sqrt{64 \beta I_{o} / 3} \\
b_{\sigma}=h_{\sigma} \sqrt{\beta / \delta} & b_{w}=h_{w} \sqrt{\beta /(3 \delta)} \\
t_{f \sigma}=\delta b_{\sigma} & t_{f w}=\delta b_{w} \\
I_{x \sigma}=2 \beta h_{\sigma}^{4} / 3 & I_{x w}=\beta h_{w}^{4} / 3 \\
W_{x \sigma}=4 \beta h_{\sigma}^{3} & W_{x w}=2 \beta h_{w}^{3} / 3
\end{array}
$$

\section{Numerical data}

$$
p=90 \mathrm{~N} / \mathrm{mm}, L=15 \mathrm{~m}, f_{y}=235 \mathrm{MPa}, f_{y l}=f_{y} / 1.1=213.6 \mathrm{MPa} .
$$

\subsection{Optimum design of unprotected beam with stress constraint}

Factored load in ambient temperature

$$
p_{\sigma}=1.5 p+1.1 \rho_{1} A_{\sigma}
$$

Factored load in fire

$$
p_{\sigma f i}=1.5 p+1.1 \rho_{1} A_{\sigma f i} ; A_{\sigma f i}=4 \beta_{f i} h_{\sigma}^{2}
$$

Bending moment for fire

$$
M_{f i}=p_{\sigma f i} L^{2} / 8
$$

Bending moment capacity in ambient temperature

$$
M_{0}=W_{x \sigma} f_{y 1}
$$

The utilization factor

$$
\mu_{0}=\frac{M_{f i}}{M_{0}}
$$

The ratio of perimeter/cross-section area for a box beam 


$$
\frac{A_{m}}{V}=\frac{2\left(h_{\sigma}+b_{\sigma}\right)}{A_{\sigma}}=\frac{1+\sqrt{\frac{\beta}{\delta}}}{2 \beta_{f i} h_{\sigma}}=\frac{61.41 \varepsilon \alpha_{f i}}{h_{\sigma}}
$$

The search for the optimum $h_{\sigma}$ is performed according to section 2 using the critical temperature method. The result for fire resistance time $R=30 \mathrm{~min}$ is $h_{\sigma}=1230 \mathrm{~mm}$. The other data for the beam are given in Table 4.

The maximum stress

$$
\sigma_{\max \sigma}=\frac{M_{f i}}{W_{x \sigma}}
$$

where $W_{x \sigma}$ is calculated according to eqn. (38).

The maximum deflection

$$
w_{\max \sigma}=\frac{5 p_{w f i \sigma} L^{4}}{384 k_{E \Theta} E I_{x \sigma}}
$$

where $k_{E \Theta}$ is calculated according to eqn. (27),

$$
p_{w f i \sigma}=p+\rho_{1} A_{\sigma}
$$

For the cost calculation eqns. (32)-(35) are used with the following changes:

$$
V_{\sigma}=A_{\sigma} L ; S_{\sigma}=2 L\left(h_{\sigma}+b_{\sigma}\right), \quad a_{w \sigma}=0.3 \frac{t_{w \sigma}}{2}
$$

The costs are calculated similar to eqns. $(32,33,34)$ with the following differences:

$$
K_{m}=k_{m} \rho V_{\sigma}, \quad K_{p}=k_{p} S_{\sigma}
$$

\subsection{Optimum design of the protected beam with stress constraint}

The optimization is performed using Table 3. Thus, subscripts of $\sigma 1$ are used. The optimum height of the beam is $h_{\sigma l}=990 \mathrm{~mm}$.

$$
\begin{aligned}
& \sigma_{\max \sigma 1}=\frac{p_{\sigma 1} L^{2}}{8 W_{x \sigma 1}} ; p_{\sigma 1}=1.5 p+1.1 \rho_{1} A_{\sigma 1} \\
& w_{\max \sigma 1}=\frac{5 p_{w \sigma 1} L^{4}}{384 E I_{x \sigma 1}} ; p_{w \sigma 1}=p+\rho_{1} A_{\sigma 1}
\end{aligned}
$$

The costs are calculated similar to section 3.6.

It should be mentioned that the self mass of the protection can be neglected. (The specific mass of plasterboard protection Rigips of thickness $12.5 \mathrm{~mm}$ is $10.5 \mathrm{~kg} / \mathrm{m}^{2}$, and that of an intumescent painting of thickness $2 \mathrm{~mm}$ is $3.5 \mathrm{~kg} / \mathrm{m}^{2}$.)

The results are given in Table 4. 
Table 4. Results for unprotected and protected beams with stress constraint. Dimensions in mm, stresses in $\mathrm{MPa}$, costs in $\$$

\begin{tabular}{cc}
\hline Unprotected & Protected \\
\hline$h_{\sigma}=1230$ & $h_{\sigma l}=990$ \\
$b_{\sigma}=960$ & $b_{\sigma l}=775$ \\
$t_{w \sigma}=60$ & $t_{w \sigma l}=30$ \\
$t_{f \sigma}=38$ & $t_{f \sigma l}=19$ \\
$\sigma_{\max \sigma}=69$ & $\sigma_{\max \sigma l}=202$ \\
$w_{\max \sigma}=22$ & $w_{\max \sigma}=31$ \\
$K_{m}=17280$ & $K_{m l}=6965$ \\
$K_{w}=2670$ & $K_{w l}=870$ \\
$K_{p}=1892$ & $K_{\text {pro }}=3177, K_{\text {prol }}=476$ \\
$K=21840$ & $K_{l}=11010, K_{2}=8311$ \\
\hline
\end{tabular}

Results in Table 4 show that the protected beam is much more cheaper than the unprotected one. The protection with plasterboard Rigips is cheaper than the Polylack painting.

\subsection{Optimum design of unprotected beam with deflection constraint}

Formulae in the right side column of Table 3 with subscript $w$ are used. Eqns. (52)-(55) are used with subscript $w$ instead of $\sigma$. eqn. (56) is changed to

$$
\mu_{0}=\frac{p_{w f i}}{p_{w}}=\frac{E_{f i} I_{0 f i}}{E I_{0}}=\frac{k_{E \Theta}\left(600^{0}\right)}{\beta_{1}}=\frac{0.31}{0.6}=0.517
$$

Eqn. (57) is changed to

$$
\frac{A_{m}}{V}=\frac{2\left(h_{w}+b_{w}\right)}{A_{w}}=\frac{3\left(1+\sqrt{\frac{\beta}{3 \delta}}\right)}{\beta h_{w}}=\frac{75.06 \varepsilon \alpha_{f i}}{h_{w}}
$$

The critical temperature according to eqn. (8) is $579^{\circ} \mathrm{C}$.

The optimum design procedure according to section 2 for fire resistant time $R=30$ min results in $h_{\text {wopt }}=$ $1500 \mathrm{~mm}$.

In eqns. (58)-(62) the subscripts $\sigma$ are changed to $w$.

The optimum beam dimensions and characteristics are summarized in Table 5. 


\subsection{Optimum design of the protected beam with deflection constraint}

The optimization is performed using Table 3. Thus, subscripts $w 1$ are used. The optimum height of the beam is $h_{w l}=1050 \mathrm{~mm}$. In eqns. (63) and (64) subscripts $\sigma 1$ are changed to $w 1$.

Results are given in Table 5.

Table 5. Results for unprotected and protected beams with deflection constraint. Dimensions in mm, stresses in $\mathrm{MPa}$, costs in $\$$

\begin{tabular}{cc}
\hline Unprotected & Protected \\
\hline$h_{w}=1500$ & $h_{w l}=1050$ \\
$b_{w}=680$ & $b_{w l}=475$ \\
$t_{w w}=74$ & $t_{w w l}=32$ \\
$t_{f w}=27$ & $t_{f w l}=19$ \\
$\sigma_{\text {maxw }}=75$ & $\sigma_{\operatorname{maxwl}}=255$ \\
$w_{\text {maxw }}=21$ & $w_{\operatorname{maxwl}}=37$ \\
$K_{m}=17390$ & \\
$K_{w}=3789$ & \\
$K_{p}=1884$ & \\
$K=23070$ & \\
\hline
\end{tabular}

It can be seen that the protected beam does not fulfil the stress constraint $(255>213 \mathrm{MPa})$, thus the costs are not calculated for this case.

Comparison of Tables 4 and 5 shows that the deflection constraint results in a more expensive beam than that with the stress constraint.

\section{Conclusions}

A compressed rod of welded square box cross-section is designed for overall and local buckling. In the case of a simply supported beam of welded box section loaded by bending and shear the stress, deflection and local buckling constraints are considered.

In the optimization procedure the systematic search method is used with MathCad program. In the case of bent box beam the optimum dimensions are derived by an analytical method.

The cost function consists of the cost of material, assembly, painting and fire protection. Two types of protection are considered: intumescent painting Polylack and plasterboard Rigips. 
The fire resistance time is $30 \mathrm{~min}$ for unprotected and $60 \mathrm{~min}$ for protected structures. The critical temperature method is suitable for the design using formulae given by Eurocode 3.

In the case of bent beam the structure for stress constraint is cheaper than that for deflection constraint. In both structures the protected ones are much more cheaper than the unprotected ones. This difference is caused by the significant difference of section thicknesses.

\section{Acknowledgements}

The research was supported by the National Office for Research and Technology (NKTH) through a bilateral Hungarian-Romanian co-operation (RO 06/2007). This activity is financed by the Research and Technology Innovation Fund. The project number is OMFB-00591/2008. The research was supported also by the Hungarian Scientific Research Fund within the project OTKA T 75678, which is gratefully acknowledged.

\section{References}

[1] Farkas,J. and Jármai,K. Analysis and optimum design of metal structures, Rotterdam: Balkema, 1997.

[2] Farkas,J. and Jármai,K. Economic design of metal structures, Rotterdam: Millpress, 2003.

[3] Farkas,J. and Jármai,K. Design and optimization of metal structures, Chichester: Horwood Publishing Ltd., 2008.

[4] Eurocode 1: Actions on structures. Part 1-2. General actions. Actions on structures exposed to fire. Brussels, 2005.

[5] Eurocode 3: Design of steel structures. Part 1-2. General rules. Structural fire design. Brussels, 2005.

[6] Eurocode 3. Design of steel structures. Part 1-1. General structural rules. Brussels, 2005.

[7] Knobloch,M., Fontana,M. and Frangi,A.: On the interaction of global and local buckling of square hollow sections in fire, in: $5^{\text {th }}$ Internat. Conference on coupled instabilities in metal structures. CMIS 2008. Sydney, Australia. pp. 587-594.

[8] Heidarpour,A. and Bradford,M.A. Local buckling and slenderness limits for flange outstands at elevated temperatures, Journal of Constructional Steel Research, 2007, 63(5), 591-598.

[9] Personal communication, Dunamenti Fire Protection Co. Febr. 2009, http://www.dunamenti.hu/index_en_new.php

[10] Rigips pricelist, http://www.fatelep.ich.hu/gipszkarton-rigips-profil.html 